\title{
Development of microstructure and fracture toughness of AL-6063 alloy using equal channel angular extrusion
}

\begin{abstract}
Severe plastic deformation (SPD) is one of the processes used to refine the microstructure of materials. Equal Channel Angular Extrusion (ECAE) is the most common method of SPD. In this study, AL-6063grain refinement was performed using the Equal Channel Angular Extrusion method. The material was extruded up to 6 passes at a temperature of $200^{\circ} \mathrm{C}$ following route $\mathrm{A}$. A suitable die sets equipped with heating elements alongside a thermometer was employed with the intention of extruding the material. Between the two channels, there was an angle of $90^{\circ}$. The results showed that the material grain average diameter decreased from $45 \mathrm{\varepsilon m}$ to $2.8 \mathrm{\varepsilon m}$ after 6 passes of the ECAE. The Charpy test was used for measuring the fracture energy of various samples. The fracture toughness was the function of this energy. According to this experiment, the fracture toughness rose as the number of the ECAE process elevated. Moreover, the toughness improved for $90 \%$ after 6 passes of the ECAE process.
\end{abstract}

Keyword: AL-6063; ECAE; Fracture toughness; Grain size; Microstructure 